\title{
Metabolomics in Cancer Biomarker Research
}

\author{
Xiaoyan Wang ${ }^{1} \cdot$ Shaoqiu Chen ${ }^{1} \cdot$ Wei Jia ${ }^{2}$ (I)
}

Published online: 11 November 2016

(C) Springer International Publishing AG 2016

\begin{abstract}
Purpose of Review As a research tool for quantitative measurement of small molecule metabolites, metabolomics has been devoted to search for biomarkers that can be used for diagnosis and prognosis of malignant tumors. The purpose of this review is to summarize recent literatures on metabolomicbased cancer biomarker research and to discuss the current issues and challenges in the clinical applications of metabolomics technologies.

Recent Findings This article reviews metabolomics-based cancer biomarker studies reported in the past 3-5 years, summarizes the classes of potential metabolite markers, and discusses the current problems in study designs as well as analytical platforms and methodologies.

Summary The application of metabolomics showed great potential in disease diagnosis, prognosis, and patient stratifications in most types of tumors. In the future, with improvement and standardization of the study designs and analytical protocols, metabolomics can serve as an effective tool for cancer translational research.
\end{abstract}

Keywords Metabolomics $\cdot$ Biomarkers $\cdot$ Metabolite markers $\cdot$ Diagnosis $\cdot$ Prognosis

This article is part of the Topical Collection on Pharmacometabolomics and Toxicometabolomics

Wei Jia

wjia@cc.hawaii.edu

1 Ministry of Education Key Laboratory of Systems Biomedicine, Shanghai Center for Systems Biomedicine, Shanghai Jiao Tong University, Shanghai 200240, People's Republic of China

2 University of Hawaii Cancer Center, Honolulu, HI 96813, USA

\section{Introduction}

Recent data showed that in the USA there will be $1,680,210$ new cancer patients and 595,690 cancer deaths in 2016 [1]. Cancer is a multifactorial disease involving various genetic aberrations and diet, lifestyle and environmental factors. The prognosis of the tumor depends greatly on the cancer stage and molecular subtypes at the time of diagnosis. Hence, rapid and precise detections of tumors are needed at early stages or even the precancerous stages. As endpoints of gene, transcription and protein expressions, small molecule metabolites can reflect the outcome of the gene-protein-environment interactions and thus, accurately represent the metabolic phenotypes (metabotypes) of various types of cancer [2]. The metabotypes of cancer patients can be critically important for their personalized treatment planning [3]. Metabolomics strategies enable us to discriminate between samples of cancer patients and ageand gender-matched healthy subjects in geographically close regions as well as to identify unique metabotypes of these patients, potentially allowing for early diagnosis and personalized treatment [4]. This review will focus on metabolomicsbased biomarker discovery studies for cancer diagnosis and prognosis over the past $3-5$ years and will discuss the current issues and challenges in the clinical applications of metabolomics technologies.

\section{The Application of Metabolomic Strategies in Cancer Study}

Most of cancer cases are not diagnosed timely and adequately until symptoms are apparent. Medical imaging is a commonly used screening method but has massive limitations in early diagnosis and prevention [5]. Metabolomics has been employed to identify meaningful tumor markers by crosssectional comparison of case-control samples and has 
generated a great number of potential markers for the diagnosis and prognosis of many types of cancer at different stages.

\section{Applications of Metabolomics in Cancer Research: Potential Biomarkers for Diagnosis and Prognosis}

Biomarker, a measurable indicator of a biological condition, needs to possess characteristics of quantitative detection, high sensitivity, and high specificity [6]. Different from traditional biomarkers such as biochemical indices and/or protein-based markers, metabolite markers rely on analytical methods [7]. Metabolomics uses analytical instruments such as NMR, GC/ MS, and LC/MS to profile metabolites present in clinical samples such as blood, urine, stool, and tumor tissue, which identifies key metabolite markers capable of discriminating among different groups through multivariate statistical methods [7, 8]. In most of cases, the key metabolite markers identified in the first study are verified with clinical samples collected from independent patient cohorts, to test the reproducibility and validity of the metabolomics method. If the verification result fulfills certain criteria, the metabolites are regarded as a potential biomarker [9]. Most of the metabolomics biomarker studies published to date are not extensively and rigorously validated due to the fact that an independent metabolomics lab may use different platforms (NMR, GC/MS, and LC/MS) and also the difficulty in recruiting patients under the same protocols [10].

\section{Different Classes Metabolites as Potential Cancer Biomarkers}

To date, a number of metabolite markers were reported from various clinical metabolomics studies, being developed or integrated as potential diagnostic modalities. According to the Warburg effect, many metabolites in glycolysis pathway was found to be closely associated with the cell proliferation, cancer growth, and metastasis [11, 12]. Significant changes in carbohydrate metabolism in blood were revealed by metabolomics studies in patients with ovarian cancer and acute myeloid leukemia (AML) $[13,14]$. One representative "oncometabolite" comprehensively studied is 2 - hydroxyglutarate (2-HG), a product of IDH1/IDH2 mutations, which was identified in many kinds of cancer including AML, breast cancer, renal cancer, intrahepatic cholangiocarcinoma, papillary thyroid carcinoma, etc. [15-22]. High level 2-HG in blood was also reported in AML and breast cancer associated with poor prognosis, suggesting that it can also serve as an effective prognostic marker $[15,23]$. In studies of oncotargets and tumor inhibitors, 2-HG was proven to suppress Par-4 transcription in vitro via inhibition of promoter activity and enhance mRNA degradation in glioma stem cells [24]. D-2HG rapidly inhibited platelet aggregation and blood clotting via a novel calcium-dependent and methylation-independent mechanism in gliomas [25]. An inhibitor of mutant IDH1 was reported to lower $2 \mathrm{HG}$ levels in vitro suggesting that IDH1 may be a promising therapeutic target in human AML cells [26]. Studies concerning mutant IDH1/IDH2 have been accelerating recently. As a result, $2-\mathrm{HG}$, the popular oncometabolite, is expected to become an efficient tumor biomarker in clinical diagnosis in the future. Table 1 lists the reports of 2-HG as a biomarker in metabolomic studies of various types of cancer.

Fatty acids are another group of metabolites associated with carcinogenesis. Serum levels of unsaturated free fatty acids were revealed to be diagnostic indicators of early-stage colorectal cancer [27]. A nutrition intervention study reported higher intakes/blood levels of the Omega-3 fatty acids, eicosapentaenoic acid (EPA), and docosahexaenoic acid (DHA), relative to the Omega-6 arachidonic acid (AA) are associated with reduced breast cancer risk [28]. Results from a mechanistic study suggest that blocking lipid synthesis could inhibit tumor re-growth and metastasis after the antiangiogenic therapy withdrawal [29].

Amino acids, a group of important molecules maintaining the physiological state of the biological systems, also play important roles in cancer development [30, 31]. Cancer cell study revealed novo serine and glycine metabolism act as necessary resources of cancer cell metastasis and malignancy [32]. Also, glycine catabolism was found to associate with rapid cancer cell proliferation [33]. Notably, as a result of the caloric restriction, glycine and serine dietary restriction can inhibit progress of cancer cells [34]. There are particularly
Table 1 A list of the reports of 2$\mathrm{HG}$ in various types of cancer

\begin{tabular}{llccrc}
\hline Diseases & Serum & Tissue & Urine & Cell line & Reference \\
\hline Acute myeloid leukemia (prognosis) & $\mathrm{Up}$ & & & {$[15]$} \\
$\begin{array}{l}\text { Triple-negative breast cancer } \\
\text { Renal cell carcinoma }\end{array}$ & & $\mathrm{Up}$ & & {$[17]$} \\
Intrahepatic cholangiocarcinoma & $\mathrm{Up}$ & & $\mathrm{Up}$ & {$[18]$} \\
Papillary thyroid carcinoma & & $\mathrm{Up}$ & & {$[19]$} \\
Breast cancer & & $\mathrm{Up}$ & $\mathrm{Up}$ & & {$[20]$} \\
Experimental colorectal neoplasia & $\mathrm{Up}$ & $\mathrm{Up}$ & & {$[21]$} \\
Breast cancer (prognosis) & & $\mathrm{Up}$ & & {$[22]$} \\
\end{tabular}


results of diagnostic significance. A recent metabolomics study showed that lowered aspartate in blood is a metabolic feature of human breast cancer [30]. Branched-chain amino acids including leucine, isoleucine, and valine, were found at high levels in blood in patients with human pancreatic adenocarcinoma [35].

Bile acids, the main components of bile, were recently discovered to play important roles in tumor regulation [36, 37]. A study reported the intrahepatic retention of hydrophobic bile acids including deoxycholate, taurocholate, taurochenodeoxycholate, and taurolithocholate that were substantially increased in the liver and blood of streptozotocin and high fat diet induced hepatocellular carcinoma mouse model. Several hydrophobic bile acids may collaboratively promote liver carcinogenesis [36].

\section{Metabolite Panels for Cancer Diagnosis and Prognosis}

The high-throughput profiling of the metabolomics likely to produce a group or a panel of metabolites with characteristic changes, high sensitivity and specificity (usually $\geq 80 \%$ ), suitable for cancer diagnosis or prognosis as a marker panel rather than a single metabolite marker $[14,38,39]$. A research project showed that a combination of C16:1, C18:2, C20:4, and $\mathrm{C} 22: 6$ has excellent diagnostic performance in serum to differentiate early-stage colorectal cancer patients from healthy controls and benign colorectal disease patients, with an AUC of 0.926 , a sensitivity of $84.6 \%$, and a specificity of $89.8 \%$ [27]. Another colorectal cancer prognosis study reported a panel of 15 significantly altered metabolites in cancer tissue with high ability to predict the rate of recurrence and survival for patients after surgery and chemotherapy. Numerous patients from different centers could share the metabolic signature despite their varied genetic background, mutations, and pathologic stages [38].

Some cancers, such as pancreatic cancer (PC), are often diagnosed at late stages, when the disease is nearly incurable. So, any forms of early diagnosis is urgently needed and of great significance. A recent metabolomics study identified a panel of five metabolites including glutamate, choline, 1,5anhydro-d-glucitol, betaine, and methylguanidine in plasma, which collectively can distinguish PC patients $(n=100)$ from healthy controls $(n=100)$ in CT (Connecticut), USA, with high sensitivity $(97.7 \%)$ and specificity $(83.1 \%)$. This panel of metabolites was then tested in an independent cohort including PC patients $(n=100)$ and controls $(\mathrm{n}=100)$ in SH (Shanghai), China, yielding satisfactory accuracy (AUC = $0.835 ; 95 \% \mathrm{CI}=0.777-0.893$ ), with a sensitivity of $77.4 \%$ and specificity of $75.8 \%$. These metabolite markers consistently and differentially expressed in both $\mathrm{CT}$ and $\mathrm{SH}$ cohorts show promise as biomarkers for early detection of PC [40].

Larger sample sizes increase the precision and validity of the biomarkers to be identified. A panel of six serum metabolite markers of glucose metabolism was identified in 400 AML patients and 446 healthy controls and tested in other six centers of China, demonstrating prognostic value in cytogenetically normal AML patients. In vitro results demonstrated enhanced glycolysis contributing to decreased sensitivity to anti-leukemic agent arabinofuranosyl cytidine (Ara-C), whereas inhibition of glycolysis suppressed AML cell proliferation and potentiated cytotoxicity of Ara-C [14].

\section{Challenges of Metabolomics in Biomarker Discovery Research}

Although metabolomics approaches are increasingly used to screening the characteristic metabolic changes of different malignant tumors and as a result, a growing number of biomarker candidates have been identified; few metabolite markers have been fully developed and translated into clinical applications [10]. There are many reasons, including problems inherent in analytical and statistical methods, and issues related to study protocols such as study design, clinical sample collections, quality controls, and data analysis and interpretation.

\section{Challenges in Clinical Sample Pretreatment and Collection}

A great number of metabolites alter as responses to the process of genetic mutation, cell proliferation, and tumor metastasis [41]. The time of sample collection during the carcinogenic process will determine rates of metabolite alteration with high diagnostic value. In early years, complete and detailed demographic, dietary, and clinical information on study participants was often lacking or not available, resulting in false positive or false negative results. So, it is not surprising that results generated from many similar studies are inconsistent with or even opposite to each other [10]. Some false results came from interferences of other conditions when there was no effective screening designed in patient recruitment beforehand. Furthermore, compared with samples of experimental animals where metabolism is relatively stable, clinical biospecimens from human were more susceptible to influence by diet, nutrition, and lifestyle factors such as smoking and drinking, and particularly the gut microbiota [42]. Hence, well-defined casecontrol studies as well as longitudinal cohort studies with complete patient information should be encouraged. Standardized procedures are of central importance to address issues in profiling methods as well as in sample collection and storage $[4,43]$. Additionally, specificity can be a controversial issue in disease diagnosis using simple metabolites, probably because various tumors may undergo metabolic transformation taking a shared set of pathways [44]. Compared with single markers, metabolite panel might be a better solution but a panel with too many metabolites may bring in technical challenges in data analysis and ascertaining the correct values in tumor detection [45]. 


\section{Challenges in Analytical Methods}

Profiling of a large array of small molecule endogenous metabolites often depends on analytical instruments and technologies that were originally designed for simple compound analysis or non-complex biological samples [46]. Compared with a small number of analytes in chemical research, human samples such as blood and urine are much more complex and unstable due to the presence of metabolic enzymes and proteins. The matrix effect of these samples are significant and will significantly impact the response of analytical instrument such as mass spectrometry. Another problem is the diverse platform technologies that are routinely used in metabolomics studies. Numerous kinds of mass spectrometry from different vendors, such as Agilent, Thermo Scientific, Applied Biosystems, Waters, Perkin Elmer, and many more, are currently being used in metabolomics labs, but have never employed a data processing software that can be shared with each other and never used a uniform database for metabolite annotation that can be cross-validated with each other [47]. Take GC-MS-based metabolomics platform as example, even with the relatively standard database, such as NIST, Mainlib, or other public or commercial database, the compound annotation is far from being accurate without reference standards [48]. Data integration and reproducibility have been highly variable among different instrument platforms, let alone the metabolite annotation from unreliable methods.

A new metabolomic approach, metabolic flux analysis, targets a specific metabolic pathway and provides a dynamic picture of metabolic changes, at cellular level, in response to a genetic modification or intervention. Specific metabolomics technologies for detection of altered metabolic flux and intracellular metabolic rate are now being rapidly developed, aiming to generate novel markers for cancer detection and novel therapeutic targets for metabolism-based cancer treatment $[49,50]$.

\section{Conclusions}

Metabolomics as a high throughput and sensitive profiling tool continues to discover promising candidate biomarkers of high value for cancer detection. With the continuous development and standardization of profiling technologies, more and more metabolomic discoveries are expected to be transformed into clinical cancer biomarkers.

Acknowledgments This work was financially supported by the National Basic Research Program of China (2012CB910102, 2007CB914700).

\section{Compliance with Ethical Standards}

Human and Animal Rights and Informed Consent This article does not contain any studies with human or animal subjects performed by any of the authors.

\section{References}

1. Siegel RL, Miller KD, Jemal A. Cancer statistics, 2016. CA Cancer J Clin. 2016;66(1):7-30. doi:10.3322/caac.21332.

2. Nath A, Chan C. Genetic alterations in fatty acid transport and metabolism genes are associated with metastatic progression and poor prognosis of human cancers. Scientific reports. 2016;6:18669. doi:10.1038/srep18669.

3. Ladep NG, Dona AC, Lewis MR, Crossey MM, Lemoine M, Okeke E, et al. Discovery and validation of urinary metabotypes for the diagnosis of hepatocellular carcinoma in West Africans. Hepatology. 2014;60(4):1291-301. doi:10.1002/hep.27264.

4. Roux A, Thevenot EA, Seguin F, Olivier MF, Junot C. Impact of collection conditions on the metabolite content of human urine samples as analyzed by liquid chromatography coupled to mass spectrometry and nuclear magnetic resonance spectroscopy. Metabolomics. 2015;11(5):1095-105. doi:10.1007/s11306-014-0764-5.

5. Mohammadzadeh Z, Safdari R, Ghazisaeidi M, Davoodi S, Azadmanjir Z. Advances in optimal detection of cancer by image processing; experience with lung and breast cancers. Asian Pacific journal of cancer prevention : APJCP. 2015;16(14):5613-8.

6. Van Gestel CA, Van Brummelen TC. Incorporation of the biomarker concept in ecotoxicology calls for a redefinition of terms. Ecotoxicology. 1996;5(4):217-25. doi:10.1007/BF00118992.

7. Qiu Y, Cai G, Su M, Chen T, Zheng X, Xu Y, et al. Serum metabolite profiling of human colorectal cancer using GCTOFMS and UPLC-QTOFMS. J Proteome Res. 2009;8(10): 4844-50. doi:10.1021/pr9004162.

8. Qiu Y, Cai G, Su M, Chen T, Liu Y, Xu Y, et al. Urinary metabonomic study on colorectal cancer. J Proteome Res. 2010;9(3):1627-34. doi:10.1021/pr901081y.

9. Chen T, Xie G, Wang X, Fan J, Qiu Y, Zheng X, et al. Serum and urine metabolite profiling reveals potential biomarkers of human hepatocellular carcinoma. Molecular \& Cellular Proteomics: MCP. 2011;10(7):M110 004945. doi:10.1074/mcp.M110.004945.

10. Ni Y, Xie G, Jia W. Metabonomics of human colorectal cancer: new approaches for early diagnosis and biomarker discovery. J Proteome Res. 2014;13(9):3857-70. doi:10.1021/pr500443c.

11. Kishton RJ, Barnes CE, Nichols AG, Cohen S, Gerriets VA, Siska $\mathrm{PJ}$, et al. AMPK is essential to balance glycolysis and mitochondrial metabolism to control T-ALL cell stress and survival. Cell Metab. 2016;23(4):649-62. doi:10.1016/j.cmet.2016.03.008.

12. Warburg O, Wind F, Negelein E. The metabolism of tumors in the body. The Journal of general physiology. 1927;8(6):519-30.

13. Cheng Y, Li L, Zhu B, Liu F, Wang Y, Gu X, et al. Expanded metabolomics approach to profiling endogenous carbohydrates in the serum of ovarian cancer patients. J Sep Sci. 2016;39(2):316-23. doi:10.1002/jssc. 201500964.

14. Chen WL, Wang JH, Zhao AH, Xu X, Wang YH, Chen TL, et al. A distinct glucose metabolism signature of acute myeloid leukemia with prognostic value. Blood. 2014;124(10): 1645-54. doi:10.1182/blood-2014-02-554204.

15. Wang JH, Chen WL, Li JM, Wu SF, Chen TL, Zhu YM, et al. Prognostic significance of 2-hydroxyglutarate levels in acute myeloid leukemia in China. Proc Natl Acad Sci U S A. 2013;110(42): 17017-22. doi:10.1073/pnas.1315558110.

16. Dang L, White DW, Gross S, Bennett BD, Bittinger MA, Driggers EM, et al. Cancer-associated IDH1 mutations produce 2-hydroxyglutarate. Nature. 2010;465(7300):966. doi:10.1038/nature09132.

17. Kanaan YM, Sampey BP, Beyene D, Esnakula AK, Naab TJ, Ricks-Santi LJ, et al. Metabolic profile of triple-negative breast cancer in African-American women reveals potential biomarkers of aggressive disease. Cancer genomics \& proteomics. 2014;11(6):279-94. 
18. Shim EH, Livi CB, Rakheja D, Tan J, Benson D, Parekh V, et al. L-2-Hydroxyglutarate: an epigenetic modifier and putative oncometabolite in renal cancer. Cancer discovery. 2014;4(11): 1290-8. doi:10.1158/2159-8290.CD-13-0696.

19. Borger DR, Goyal L, Yau T, Poon RT, Ancukiewicz M, Deshpande $\mathrm{V}$, et al. Circulating oncometabolite 2-hydroxyglutarate is a potential surrogate biomarker in patients with isocitrate dehydrogenase-mutant intrahepatic cholangiocarcinoma. Clinical cancer research : an official journal of the American Association for Cancer Research. 2014;20(7):1884-90. doi:10.1158/1078-0432.CCR-13-2649.

20. Rakheja D, Boriack RL, Mitui M, Khokhar S, Holt SA, Kapur P. Papillary thyroid carcinoma shows elevated levels of 2hydroxyglutarate. Tumour biology: the journal of the International Society for Oncodevelopmental Biology and Medicine. 2011;32(2):325-33. doi:10.1007/s13277-010-0125-6.

21. Fathi AT, Sadrzadeh H, Comander AH, Higgins MJ, Bardia A, Perry A, et al. Isocitrate dehydrogenase 1 (IDH1) mutation in breast adenocarcinoma is associated with elevated levels of serum and urine 2-hydroxyglutarate. Oncologist. 2014;19(6):602-7. doi:10.1634/theoncologist.2013-0417.

22. Montrose DC, Zhou XK, Kopelovich L, Yantiss RK, Karoly ED, Subbaramaiah K, et al. Metabolic profiling, a noninvasive approach for the detection of experimental colorectal neoplasia. Cancer Prev Res. 2012;5(12):1358-67. doi:10.1158/1940-6207.CAPR-12-0160.

23. Terunuma A, Putluri N, Mishra P, Mathe EA, Dorsey TH, Yi M, et al. MYC-driven accumulation of 2-hydroxyglutarate is associated with breast cancer prognosis. J Clin Invest. 2014;124(1):398412. doi:10.1172/JCI71180.

24. Liu Y, Gilbert MR, Kyprianou N, Rangnekar VM, Horbinski C. The tumor suppressor prostate apoptosis response-4 (Par-4) is regulated by mutant IDH1 and kills glioma stem cells. Acta Neuropathol. 2014;128(5):723-32. doi:10.1007/s00401-014-1334-7.

25. Unruh D, Schwarze SR, Khoury L, Thomas C, Wu M, Chen L, et al. Mutant IDH1 and thrombosis in gliomas. Acta Neuropathol. 2016. doi:10.1007/s00401-016-1620-7.

26. Chaturvedi A, Araujo Cruz MM, Jyotsana N, Sharma A, Yun H, Gorlich K, et al. Mutant IDH1 promotes leukemogenesis in vivo and can be specifically targeted in human AML. Blood. 2013;122(16):2877-87. doi:10.1182/blood-2013-03-491571.

27. Zhang Y, He C, Qiu L, Wang Y, Qin X, Liu Y, et al. Serum unsaturated free fatty acids: a potential biomarker panel for early-stage detection of colorectal cancer. J Cancer. 2016;7(4):477-83. doi:10.7150/jca.13870.

28. Fabian CJ, Kimler BF, Phillips TA, Nydegger JL, Kreutzjans AL, Carlson SE, et al. Modulation of breast cancer risk biomarkers by high-dose omega-3 fatty acids: phase II pilot study in postmenopausal women. Cancer Prev Res. 2015;8(10):922-31. doi:10.1158/1940-6207.CAPR-14-0336.

29. Sounni NE, Cimino J, Blacher S, Primac I, Truong A, Mazzucchelli $\mathrm{G}$, et al. Blocking lipid synthesis overcomes tumor regrowth and metastasis after antiangiogenic therapy withdrawal. Cell Metab. 2014;20(2):280-94. doi:10.1016/j.cmet.2014.05.022.

30. Xie G, Zhou B, Zhao A, Qiu Y, Zhao X, Garmire L, et al. Lowered circulating aspartate is a metabolic feature of human breast cancer. Oncotarget. 2015;6(32):33369-81. doi:10.18632/oncotarget.5409.

31. Geng D, Sun D, Zhang L, Zhang W. The therapy of gefitinib towards breast cancer partially through reversing breast cancer biomarker arginine. Afr Health Sci. 2015;15(2):5947. doi:10.4314/ahs.v15i2.36.

32. Locasale JW, Grassian AR, Melman T, Lyssiotis CA, Mattaini KR, Bass AJ, et al. Phosphoglycerate dehydrogenase diverts glycolytic flux and contributes to oncogenesis. Nat Genet. 2011;43(9):86974. doi:10.1038/ng.890.
33. Jain M, Nilsson R, Sharma S, Madhusudhan N, Kitami T, Souza $\mathrm{AL}$, et al. Metabolite profiling identifies a key role for glycine in rapid cancer cell proliferation. Science. 2012;336(6084):1040-4. doi:10.1126/science.1218595.

34. Maddocks OD, Berkers CR, Mason SM, Zheng L, Blyth K, Gottlieb E, et al. Serine starvation induces stress and p53dependent metabolic remodelling in cancer cells. Nature. 2013;493(7433):542-6. doi:10.1038/nature11743.

35. Mayers JR, Wu C, Clish CB, Kraft P, Torrence ME, Fiske BP, et al. Elevation of circulating branched-chain amino acids is an early event in human pancreatic adenocarcinoma development. Nat Med. 2014;20(10):1193-8. doi:10.1038/nm.3686.

36. Xie G, Wang X, Huang F, Zhao A, Chen W, Yan J, et al. Dysregulated hepatic bile acids collaboratively promote liver carcinogenesis. Int J Cancer. 2016. doi:10.1002/ijc.30219.

37. Zang X, Jones CM, Long TQ, Monge ME, Zhou M, Walker LD, et al. Feasibility of detecting prostate cancer by ultraperformance liquid chromatography-mass spectrometry serum metabolomics. J Proteome Res. 2014;13(7):3444-54. doi:10.1021/pr500409q.

38. Qiu Y, Cai G, Zhou B, Li D, Zhao A, Xie G, et al. A distinct metabolic signature of human colorectal cancer with prognostic potential. Clinical cancer research : an official journal of the American Association for Cancer Research. 2014;20(8):2136-46. doi:10.1158/1078-0432.CCR-13-1939.

39. Kobayashi T, Nishiumi S, Ikeda A, Yoshie T, Sakai A, Matsubara A, et al. A novel serum metabolomics-based diagnostic approach to pancreatic cancer. Cancer epidemiology, biomarkers \& prevention : a publication of the American Association for Cancer Research, cosponsored by the American Society of Preventive Oncology. 2013;22(4):571-9. doi:10.1158/1055-9965.EPI-12-1033.

40. Xie G, Lu L, Qiu Y, Ni Q, Zhang W, Gao YT, et al. Plasma metabolite biomarkers for the detection of pancreatic cancer. J Proteome Res. 2015;14(2):1195-202. doi:10.1021/pr501135f.

41. Kerr EM, Gaude E, Turrell FK, Frezza C, Martins CP. Mutant Kras copy number defines metabolic reprogramming and therapeutic susceptibilities. Nature. 2016;531(7592):110 3. doi:10.1038/nature16967.

42. Sinha R, Ahn J, Sampson JN, Shi J, Yu G, Xiong X, et al. Fecal microbiota, fecal metabolome, and colorectal cancer interrelations. PLoS One. 2016;11(3):e0152126. doi:10.1371/journal.pone.0152126.

43. Abuja PM, Ehrhart F, Schoen U, Schmidt T, Stracke F, Dallmann $\mathrm{G}$, et al. Alterations in human liver metabolome during prolonged cryostorage. J Proteome Res. 2015;14(7): 2758-68. doi:10.1021/acs.jproteome.5b00025.

44. Shen Y, Wang JH, Chen WL, Jia W, Chen SJ, Chen Z. Reply to Struys: role of biomarker of 2-hydroxyglutarate in acute myeloid leukemia. Proc Natl Acad Sci U S A. 2013;110(51): E4940.

45. Fiandaca MS, Zhong X, Cheema AK, Orquiza MH, Chidambaram S, Tan MT, et al. Plasma 24-metabolite panel predicts preclinical transition to clinical stages of Alzheimer's disease. Front Neurol. 2015;6:237. doi:10.3389/fneur.2015.00237.

46. Brown MV, McDunn JE, Gunst PR, Smith EM, Milburn MV, Troyer DA, et al. Cancer detection and biopsy classification using concurrent histopathological and metabolomic analysis of core biopsies. Genome medicine. 2012;4(4):33. doi: $10.1186 / \mathrm{gm} 332$.

47. Sud M, Fahy E, Cotter D, Azam K, Vadivelu I, Burant C, et al. Metabolomics workbench: an international repository for metabolomics data and metadata, metabolite standards, protocols, tutorials and training, and analysis tools. Nucleic Acids Res. 2016;44(D1): D463-70. doi:10.1093/nar/gkv1042.

48. Tsugawa H, Bamba T, Shinohara M, Nishiumi S, Yoshida M, Fukusaki E. Practical non-targeted gas chromatography/ 
mass spectrometry-based metabolomics platform for metabolic phenotype analysis. J Biosci Bioeng. 2011;112(3):292-8. doi:10.1016/j.jbiosc.2011.05.001.

49. Tedeschi PM, Johnson-Farley N, Lin H, Shelton LM, Ooga T, Mackay G, et al. Quantification of folate metabolism using transient metabolic flux analysis. Cancer \& metabolism. 2015;3:6. doi:10.1186/s40170-015-0132-6.

50. Schwartz JM, Barber M, Soons Z. Metabolic flux prediction in cancer cells with altered substrate uptake. Biochem Soc Trans. 2015;43(6):1177-81. doi:10.1042/BST20150149. 